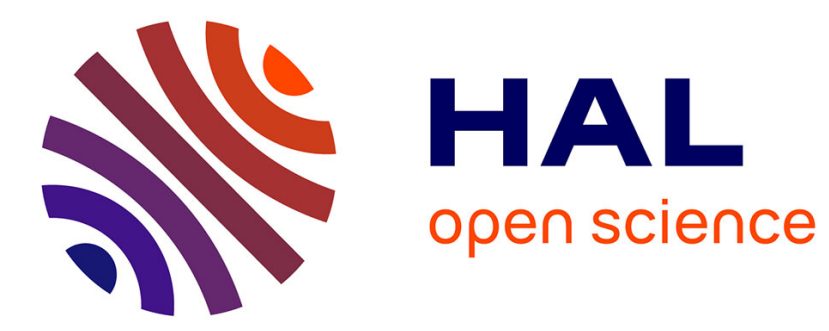

\title{
Computing heteroclinic orbits using adjoint-based methods
}

Mirko Farano, Stefania Cherubini, Jean-Christophe Robinet, P. de Palma, T. M. Schneider

\section{- To cite this version:}

Mirko Farano, Stefania Cherubini, Jean-Christophe Robinet, P. de Palma, T. M. Schneider. Computing heteroclinic orbits using adjoint-based methods. Journal of Fluid Mechanics, 2018, 858, pp.R3. 10.1017/jfm.2018.860 . hal-02454296

\section{HAL Id: hal-02454296 https://hal.science/hal-02454296}

Submitted on 24 Jan 2020

HAL is a multi-disciplinary open access archive for the deposit and dissemination of scientific research documents, whether they are published or not. The documents may come from teaching and research institutions in France or abroad, or from public or private research centers.
L'archive ouverte pluridisciplinaire HAL, est destinée au dépôt et à la diffusion de documents scientifiques de niveau recherche, publiés ou non, émanant des établissements d'enseignement et de recherche français ou étrangers, des laboratoires publics ou privés. 


\title{
Computing heteroclinic orbits using adjoint-based methods
}

\author{
M. Farano ${ }^{1,2,3,} \dagger$, S. Cherubini ${ }^{1,} \dagger$, J.-C. Robinet ${ }^{2}$, P. De Palma ${ }^{1}$ and \\ T. M. Schneider ${ }^{3,} \dagger$ \\ ${ }^{1}$ DMMM, Politecnico di Bari, Via Re David 200, 70125 Bari, Italy \\ ${ }^{2}$ DynFluid Laboratory, Arts et Metiers ParisTech, 151 Bd de 1'Hopital, 75013 Paris, France \\ ${ }^{3}$ ECPS, École Polytechnique Fédérale de Lausanne, 1015 Lausanne, Switzerland
}

Transitional turbulence in shear flows is supported by a network of unstable exact invariant solutions of the Navier-Stokes equations. The network is interconnected by heteroclinic connections along which the turbulent trajectories evolve between invariant solutions. While many invariant solutions in the form of equilibria, travelling waves and periodic orbits have been identified, computing heteroclinic connections remains a challenge. We propose a variational method for computing orbits dynamically connecting small neighbourhoods around equilibrium solutions. Using local information on the dynamics linearized around these equilibria, we demonstrate that we can choose neighbourhoods such that the connecting orbits shadow heteroclinic connections. The proposed method allows one to approximate heteroclinic connections originating from states with multi-dimensional unstable manifold and thereby provides access to heteroclinic connections that cannot easily be identified using alternative shooting methods. For plane Couette flow, we demonstrate the method by recomputing three known connections and identifying six additional previously unknown orbits.

Key words: mathematical foundations, variational methods, nonlinear dynamical systems

\section{Introduction}

The understanding of the dynamics of turbulence has been recently boosted by the adoption of a dynamical-system representation of the problem, inspired by the early work of Hopf (1948) and extended during the last 20 years to the case of turbulent shear flows. According to this dynamical view point, the time evolution of the flow corresponds to a trajectory in state space, which recurrently approaches

†Email addresses for correspondence: mirko.farano@epfl.ch, stefania.cherubini@poliba.it, tobias.schneider@epfl.ch 


\section{Farano, S. Cherubini, J. C. Robinet, P. De Palma and T. M. Schneider}

the exact invariant solutions populating the state space. Turbulence is linked to the existence of a multitude of such invariant solutions of the Navier-Stokes (NS) equations (Budanur et al. 2017), in the form of equilibria, travelling waves, periodic orbits and relative periodic orbits (Kawahara, Uhlmann \& Van Veen 2012). The transiently visited solutions capture organized flow structures, such as streaks in turbulent boundary layers, as well as puffs in pipe flows (Hof et al. 2004), and are thus also termed exact coherent states (ECSs). Due to their dynamical relevance there has been a strong recent effort to compute invariant solutions, especially for pipe (Faisst \& Eckhardt 2003; Wedin \& Kerswell 2004; Duguet, Willis \& Kerswell 2008; Willis, Cvitanović \& Avila 2013; Budanur et al. 2017) and Couette flow (Nagata 1990; Kawahara \& Kida 2001; Viswanath 2007; Gibson, Halcrow \& Cvitanović 2008; Schneider et al. 2008; Gibson, Halcrow \& Cvitanović 2009; Halcrow et al. 2009). In these two reference flow cases, the state space contains a linearly stable equilibrium solution corresponding to the laminar flow state, as well as a chaotic set supporting turbulent dynamics. Embedded in the chaotic saddle are ECSs dynamically connected by their entangled stable and unstable manifolds. The ECSs are all unstable so that a turbulent trajectory is believed to be transiently attracted to an individual ECS along its stable manifold before it leaves along the unstable manifold and is captured by another ECS. Within the emerging dynamical systems picture, turbulence can thus be understood as a walk along dynamical connections between various ECSs (Suri et al. 2017). To characterize the turbulence-supporting saddle we not only need to compute the invariant states but also identify homo- and heteroclinic connections between them. These connections form the network along which turbulent trajectories evolve between the ECSs and are thus key for understanding the turbulent dynamics. Unfortunately, finding heteroclinic connections is a very difficult task and there is no general and robust algorithm to compute them. In fact, the first heteroclinic connection between two equilibria of the three-dimensional (3D) Navier-Stokes equations for a shear flow has been reported only recently. Gibson et al. (2008) identified a connection in Couette flow at Reynolds number $R e=400$. For the same flow and parameters, two additional heteroclinic connections have been reported by the same research group (Halcrow et al. 2009). A homoclinic connection from and to the edge state in plane Couette flow was identified by Van Veen \& Kawahara (2011), who showed that this connection underlies strongly dissipative bursting events. Evidence for a likely heteroclinic connection between two relative periodic orbits for the case of pipe flow has been recently found by Hof \& Budanur (2017). A periodic-like solution (Toh \& Itano 2003) in plane Poiseuille flow suggests heteroclinic connection between a single-streak and a double-streak state. Likewise a connection between two symmetry-related travelling waves on the laminar-turbulent boundary for the asymptotic suction boundary layer is strongly suggested by Kreilos et al. (2013). These studies suggest that connections between exact invariant solutions are dynamically relevant and may underlie both bursts and puff formation. Despite their obvious importance, for full 3D shear flows the only currently available algorithms for computing heteroclinic connections are the shooting method of Halcrow et al. (2009) and the boundary-value problem approach by Van Veen, Kawahara \& Atsushi (2011).

We propose an alternative method for identifying heteroclinic connections and apply it to plane Couette flow at $R e=400$. The method entails two steps. First, we construct an orbit that connects small energy shells around two equilibrium states. This step is based on nonlinear minimization using the Lagrange multiplier method. In a second step, we use local information on the dynamics linearized around the initial and final 


\section{Adjoints for computing heteroclinic orbits}

equilibria to confirm that the constructed orbit shadows a heteroclinic connection located within the intersection of the unstable manifold of the initial and the stable manifold of the final equilibria. We first present the numerical approach and validate the method with respect to the results of Halcrow et al. (2009), obtaining the same three heteroclinic connections. Then, the proposed method is used to identify six new heteroclinic connections. Finally, we discuss advantages and limitations of the proposed method in comparison to alternative approaches for computing heteroclinic connections.

\section{Problem formulation}

We consider plane Couette flow at Reynolds number $R e=400$, where nondimensional variables are chosen to have half of the distance between the plates equal to $h=1$ and half of the difference between the velocity of the two plates equal to 1 . The problem is studied by numerically solving the Navier-Stokes equations for incompressible flow. Simulations are performed in a computational domain of streamwise and spanwise size $L_{x}=2 \pi / 1.14$ and $L_{z}=2 \pi / 2.5$, respectively (Waleffe 2003). $x, y, z$ represent the streamwise, wall-normal and spanwise coordinates, and $u, v, w$ are the three corresponding Cartesian components of the velocity vector, $\boldsymbol{u}$. Dirichlet boundary conditions for the three velocity components are imposed at the upper and lower wall $\left(u_{\text {wall }}= \pm 1\right)$, whereas periodicity is prescribed in the streamwise and spanwise directions. Computations are performed using Channelflow (Gibson 2014). For spectral discretization, we use 32 Fourier points in the $x$ and $z$ directions and 35 Chebyshev points in the $y$ direction. Both domain and discretization are identical to Halcrow et al. (2009) in order to validate our procedure with their results.

\section{Algorithm for computing heteroclinic connections}

The main idea of the present work is to employ a nonlinear variational minimization algorithm based on an iterative direct-adjoint procedure (Cherubini et al. 2010; Pringle \& Kerswell 2010; Kerswell 2018) to compute connections between finite neighbourhoods of equilibrium states and show that those orbits approximate heteroclinic connections. Alternative variational approaches have also been proposed for computing periodic orbits (Lan \& Cvitanović 2004) and heteroclinic connections in lower-dimensional problems (Dong \& Lan 2014) including two-dimensional (2D) atmospheric flow models (Crommelin 2003).

Along a heteroclinic connection, the trajectory leaves the initial equilibrium $\left(E Q_{o u t}\right)$ to approach the final equilibrium $\left(E Q_{i n}\right)$. Since it takes an infinite time to traverse the connection we approximate it by a trajectory starting in a small but finite neighbourhood of the initial equilibrium and reaching the neighbourhood of the final one. The passage time $T$ is finite and we ensure that results are robust with respect to the choice of the neighbourhoods.

Technically, we fix energy shells $E_{0_{\text {out }}}$ and $E_{0_{\text {in }}}$, around the initial and final equilibrium. For fixed passage time $T$ we find the initial perturbation $\boldsymbol{u}^{\prime}(0)=$ $\boldsymbol{u}(0)-\boldsymbol{u}_{E Q_{\text {out }}}$ constrained to $E_{0_{\text {out }}}$, such that the distance from the final equilibrium is minimized at passage time $T$. By gradually increasing the passage time, we determine the time $T$ of the first local minimum of the objective function lower than a chosen threshold $E_{0_{i n}}$.

For minimizing the distance from the final equilibrium $E(T)=\| \boldsymbol{u}^{\prime}(T)+\boldsymbol{u}_{E Q_{o u t}}-$ $\boldsymbol{u}_{E Q_{i n}} \|_{2}^{2}$, with $\|\bullet\|_{2}^{2}=1 / V \int_{V} \bullet 2 \mathrm{~d} V$, and $V$ the volume of the computational domain, 


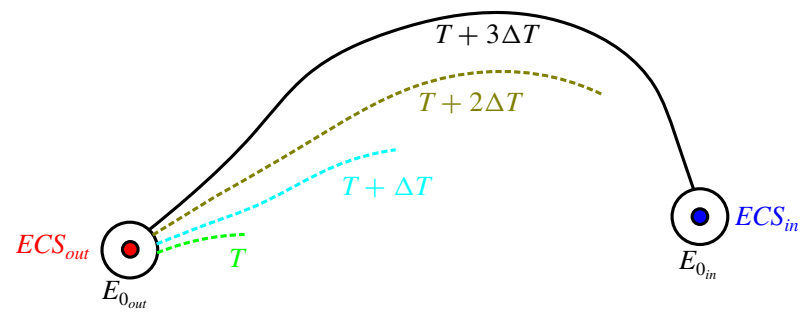

FIGURE 1. Sketch describing the CHELA algorithm: the initial condition and the related trajectory towards the final EQ change after updating the time $T$ (dashed lines). The algorithm stops when $T$ is sufficiently large to lead the flow field close enough to the final state (solid line).

we consider the Lagrangian

$$
\begin{aligned}
& \mathcal{L}\left(\boldsymbol{u}^{\prime}, p^{\prime}, \boldsymbol{u}^{\dagger}, p^{\dagger}, \boldsymbol{u}^{\prime}(T), \boldsymbol{u}^{\prime}(0), \lambda\right)=\left\|\boldsymbol{u}^{\prime}(T)+\boldsymbol{u}_{E Q_{\text {out }}}-\boldsymbol{u}_{E Q_{\text {in }}}\right\|_{2}^{2} \\
& \quad-\int_{0}^{T}\left\langle\boldsymbol{u}^{\dagger}, N S\left(\boldsymbol{u}^{\prime}, \boldsymbol{u}_{E Q_{\text {out }}}, p^{\prime}\right)\right\rangle \mathrm{d} t-\int_{0}^{T}\left\langle p^{\dagger}, \nabla \cdot \boldsymbol{u}^{\prime}\right\rangle \mathrm{d} t-\lambda\left(\left\|\boldsymbol{u}^{\prime}(0)\right\|_{2}^{2}-E_{0_{\text {out }}}\right),
\end{aligned}
$$

where $\langle\boldsymbol{a}, \boldsymbol{b}\rangle$ indicates the scalar product $1 / V \int_{V}(\boldsymbol{a} \cdot \boldsymbol{b}) \mathrm{d} V$. Setting the variation of $\mathcal{L}$ with respect to the fields $\boldsymbol{u}^{\prime}, p^{\prime}$ to zero yields adjoint equations for the Lagrange multipliers $\boldsymbol{u}^{\dagger}, p^{\dagger}$ that enforce the NS equations. The vanishing gradient with respect to $\boldsymbol{u}^{\prime}(T)$ yields the compatibility conditions at $t=T$, namely, $\boldsymbol{u}^{\dagger}(T)=\boldsymbol{u}^{\prime}(T)+\boldsymbol{u}_{E Q_{\text {out }}}-\boldsymbol{u}_{E Q_{\text {in }}}$. Integrating the NS equations from $t=0$ to $t=T$, using the compatibility condition to initialize the adjoint equations and integrating them backwards in time from $t=T$ to $t=0$ yields the gradient with respect to the initial condition $\left(\delta \mathcal{L} / \delta \boldsymbol{u}^{\prime}(0)\right)=\boldsymbol{u}(0)^{\dagger}$. Iterative gradient descent using the gradient-rotation method (Foures, Caulfield \& Schmid 2013; Farano et al. 2015) to exactly enforce the initial energy shell constraint yields the optimal initial condition.

Technically, the algorithm consists of a double nested loop: an inner direct-adjoint loop for a fixed passage time, and an outer one increasing the passage time. Finding the minimal passage time by sequentially increasing $T$ is a key element of the proposed CHELA (Computing HEteroclinic connections by using a Lagrange multiplier Algorithm) schematically presented in figure 1 and summarized in Algorithm 1. For a fixed passage time, the inner direct-adjoint loop is carried out until the relative difference of the value of $E(T)$ between successive iterations $\epsilon=\left(\left(E(T)^{n+1}-E(T)^{n}\right) / E(T)^{n}\right.$ is lower than a given threshold $\epsilon_{c r}$, where superscript $n$ indicates the number of loop iterations. For short $T$ the trajectory will remain close to the initial equilibrium and not reach the neighbourhood of the final equilibrium, see dashed lines in figure 1 . We therefore carry out the full optimization procedure for gradually increasing passage times $T$ (outer loop) until $E(T)<E_{0_{\text {in }}}$ and the trajectory reaches the neighbourhood of the final equilibrium, as indicated by the solid line in figure 1. By starting from a short passage time and increasing it, one avoids convergence problems of the direct-adjoint looping procedure stemming from chaotic dynamics that can lead to a non-smooth optimization problem at large passage times.

We identify orbits connecting finite neighbourhoods of two equilibria in finite time, while a heteroclinic connection is traversed in infinite time. Especially in view of transient growth effects discussed below, considering finite neighbourhoods may 


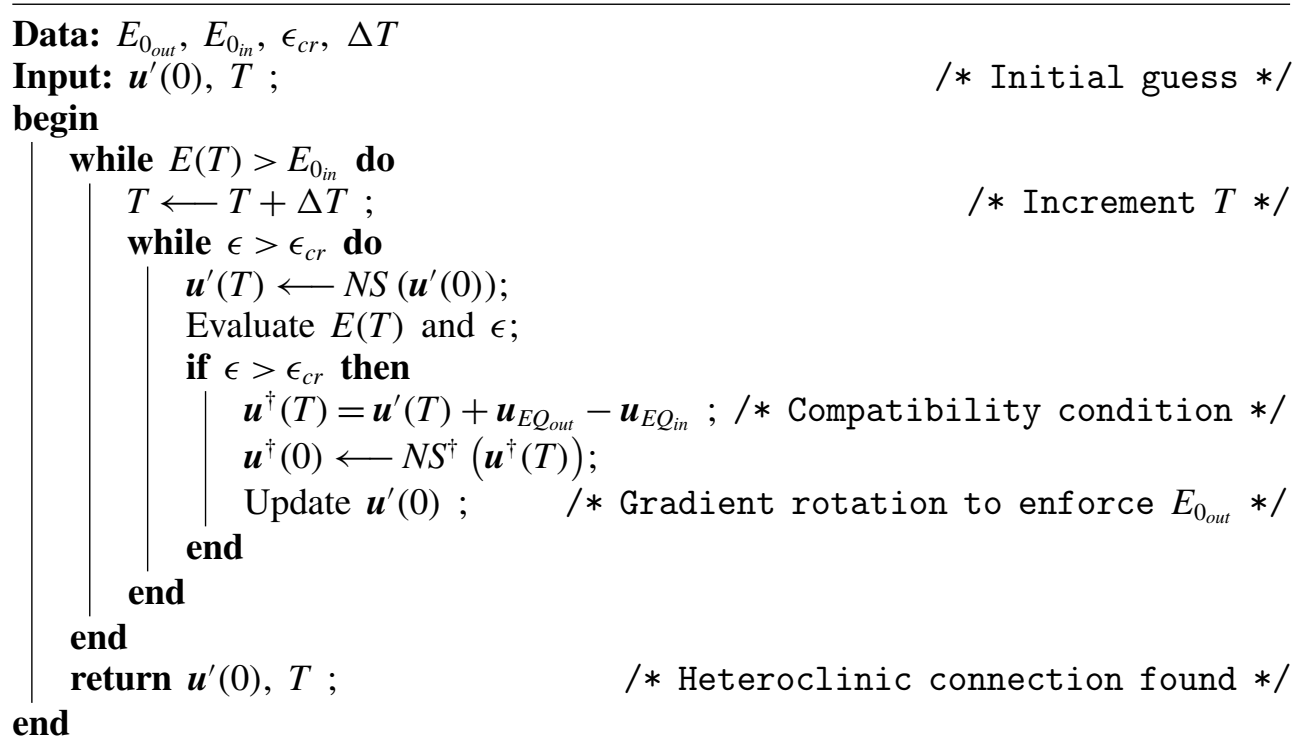

Algorithm 1: Schematic synthesis of the CHELA algorithm.

lead to spurious results and the misidentification of heteroclinic connections. By analysing the dynamics in the neighbourhood of the initial and final equilibria we thus a posteriori confirm that every connection between finite neighbourhoods leaves the initial equilibrium shadowing its unstable manifold and approaches the final equilibrium along the stable one. Together, the variational method for constructing orbits connecting neighbourhoods of equilibria and the a posteriori check that those orbits shadow the unstable (stable) manifold of the initial (final) equilibrium allows one to approximate heteroclinic connections.

\section{Results}

In this section we present approximations to nine heteroclinic connections linking seven unstable equilibrium states originally found by Halcrow et al. (2009) and available in the Channelflow database (Gibson 2014). Seven connections are found for parameters $E_{0_{\text {out }}}=10^{-8}, E_{0_{\text {in }}}=10^{-6}$ and $\epsilon_{c r}=10^{-4}$. In terms of L2-distance this corresponds to shells of radius $r_{\text {out }}=10^{-4}$ and $r_{\text {in }}=10^{-3}$. For two connections larger shells with $r_{\text {out }}=10^{-3}$ and $r_{\text {in }}=2.26 \times 10^{-3}$, corresponding to the values in Halcrow et al. (2009) are used. The larger shells reduce the passage time along the connections (underlined in table 1) and thereby avoid convergence problems of the optimization algorithm. As detailed below, we verify for all presented orbits that these shadow heteroclinic connections. First, we validated the algorithm by computing the three heteroclinic connections already found for plane Couette flow at $R e=400$ by Halcrow et al. (2009). Then, we found, for the same value of $R e$, six previously unknown heteroclinic connections.

\subsection{Connections identified and their properties}

Details, specifically the dimension of the unstable manifold of the initial equilibrium, and the passage time $T$, about the heteroclinic connections are given in table 1, where 


\begin{tabular}{lccccc}
$\begin{array}{l}\text { Heteroclinic } \\
\text { connections }\end{array}$ & Symmetry & $d\left(W^{u}\left(E Q_{\text {out }}\right)\right)$ & $d\left(W_{S}^{u}\left(E Q_{\text {out }}\right)\right)$ & $d\left(W_{S}^{u}\left(E Q_{\text {in }}\right)\right)$ & $T$ \\
$E Q 5 \rightarrow E Q 1$ & & & & & \\
$E Q 4 \rightarrow E Q 1$ & $\left(s_{1}, s_{2}, s_{3}\right)$ & 11 & 4 & 1 & 363 \\
$E Q 3 \rightarrow E Q 1$ & $\left(s_{1}, s_{2}, s_{3}\right)$ & 6 & 3 & 1 & 318 \\
$E Q 4 \rightarrow E Q 3$ & $\left(s_{1}, s_{2}, s_{3}\right)$ & 4 & 2 & 1 & 332 \\
$E Q 9 \rightarrow E Q 3$ & $\left(s_{1}, s_{2}, s_{3}\right)$ & 6 & 3 & 2 & 447 \\
$E Q 4 \rightarrow E Q 9$ & $\left(s_{3}\right)$ & 5 & 3 & 2 & 405 \\
$E Q 10 \rightarrow E Q 1$ & $\left(s_{3}\right)$ & 6 & 4 & 3 & 363 \\
$E Q 9 \rightarrow E Q 1$ & $\left(s_{3}\right)$ & 10 & 7 & 1 & 476 \\
$E Q 11 \rightarrow E Q 1$ & $\left(s_{3}\right)$ & 5 & 3 & 1 & 550 \\
\hline
\end{tabular}

TABLE 1. Computed heteroclinic connections for $R e=400$ and corresponding symmetry subspaces. The dimension of the unstable manifold of the initial equilibrium $\left(E Q_{\text {out }}\right)$ is $d\left(W^{u}\right)$, while $d\left(W_{S}^{u}\right)$ is the dimension of the intersection of the unstable manifold with the symmetry-invariant subspace reported here for the initial and final equilibrium $\left(E Q_{i n}\right)$. The last column provides the passage time for the different orbits with shell around the initial state of radius $r_{\text {out }}=10^{-4}$ ( $r_{\text {out }}=10^{-3}$ for underlined ones).

the equilibria are indicated following the same nomenclature employed by Gibson et al. (2009). The considered initial and final equilibria possess discrete symmetries which are part of the equivariance under a symmetry group. To construct a connecting trajectory within the symmetry subspace shared between initial and final equilibria, we explicitly constrain the time evolution to the shared symmetry subspace. The symmetry group considered here is $S=\left\{1, s_{1}, s_{2}, s_{3}\right\}$ and coincides with that of Gibson et al. (2008); $s_{1}$ and $s_{2}$ indicate the 'shift-reflect' and 'shift-rotate' symmetries, respectively, and $s_{3}=s_{1} s_{2}$. Four of the considered equilibria have all the symmetries in $S$, and the remaining ones have only $s_{3}$. The first three connections coincide with those described by Halcrow et al. (2009).

Two manifolds generically intersect if the sum of their dimensions is higher than or equal to the dimension of the state space they are embedded in (Halcrow et al. 2009). An intersection between the unstable manifold of the initial state and the stable one of the final state can thus generically exist if the codimension of the stable manifold in the $S$-invariant space of the final state $d\left(W_{S}^{u}\left(E Q_{i n}\right)\right)$ is smaller than or equal to the dimension of the unstable manifold of the initial state $d\left(W_{S}^{u}\left(E Q_{\text {out }}\right)\right)$. This geometric condition is satisfied for all the computed heteroclinic connections (compare the fourth and fifth columns in table 1) and indicates the structural stability of all presented connections.

The convergence histories of the computed heteroclinic connections are shown in figure 2. For validation, panel $(a)$ provides the distance of the velocity field $\boldsymbol{u}(t)$ to the target equilibrium $\boldsymbol{u}_{E Q_{i n}}$ (continuous line) and to the initial equilibrium $\boldsymbol{u}_{E Q_{o u t}}$ (dashed line) for the three connections already obtained by Halcrow et al. (2009). For a fixed passage time $T$ and time increments, $\Delta T$, chosen between 1 and 5 time units, the optimization algorithm typically requires 5 to 20 forward/backward time integrations to converge. The convergence curves are in good agreement with those obtained in the literature, with the convergence threshold achieved by Halcrow et al. (2009) indicated by a dashed line in figure $2(b)$.

Figure 2(b) shows the new six heteroclinic connections computed here. Three of the new connections lead to the edge state (Nagata 1990; Schneider et al. 2008), two of 

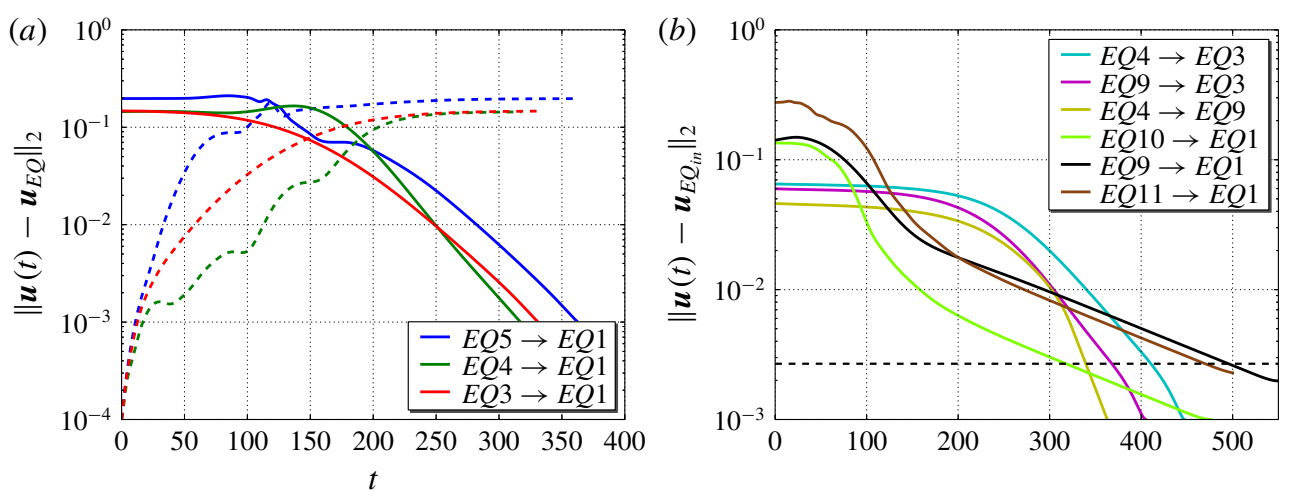

FIGURE 2. (a) Distances of the velocity field $\boldsymbol{u}(t)$ to the final equilibrium $\boldsymbol{u}_{E Q_{i n}}$ (continuous line) and to the initial one $\boldsymbol{u}_{E Q_{o u t}}$ (dashed line) along three heteroclinic connections found by Halcrow et al. (2009) versus time. (b) Distances to the final equilibrium $\boldsymbol{u}_{E Q_{i n}}$ for the new computed heteroclinic connections (continuous lines). The dashed line represents the highest residual value for the heteroclinic connections computed in Halcrow et al. (2009). The L2-norm of the considered equilibria ranges from 0.1259 to 0.4049 . (Gibson et al. 2009).

them to $E Q 3$, and one to $E Q 9$. All connections reported by Halcrow et al. (2009) land on the codimension-1 stable manifold of the edge state, which simplifies identifying those connections using a one-dimensional search algorithm. The variational method proposed here yields additional connections not involving the edge state.

\subsection{Confirming that computed orbits shadow heteroclinic connections}

Heteroclinic connections lie in the intersection of the unstable manifold of the initial equilibrium and the stable manifold of the final equilibrium. The use of finite energy shells renders the passage time along the orbit finite but may potentially lead to spurious results. A specific problem is due to transient growth effects that may allow a trajectory to leave the neighbourhood of an equilibrium along an algebraically amplified direction bypassing the unstable manifold (Farano et al. 2018). We thus analyse the dynamics close to the equilibria where curved stable and unstable manifolds can be approximated by their respective tangent spaces, the stable and unstable subspaces spanned by the stable or unstable eigenvectors of the Navier-Stokes equations linearized around the equilibrium. The analysis of the orbits connecting finite energy shells technically involves two steps: first, along the orbit we determine the range in the vicinity of both equilibria, where the dynamics is well approximated by the Navier-Stokes equations linearized around $E Q_{\text {out }}$ or $E Q_{\text {in }}$, respectively. This is achieved by explicitly comparing the nonlinear and the linear time evolution. Within the validity range of the linearization, we then project onto an orthonormal basis of the unstable tangent spaces to the invariant manifolds of both equilibria and thereby separate the energetic contributions in the stable and unstable subspaces approximating the respective manifolds. (Note that we technically only use projections on the unstable subspace spanned by the orthogonalized unstable eigenvectors. These leading eigenvectors can be accurately constructed by our iterative Arnoldi method, while most of the stable eigenvectors are challenging to compute accurately using matrix-free techniques.) 


\section{Farano, S. Cherubini, J. C. Robinet, P. De Palma and T. M. Schneider}
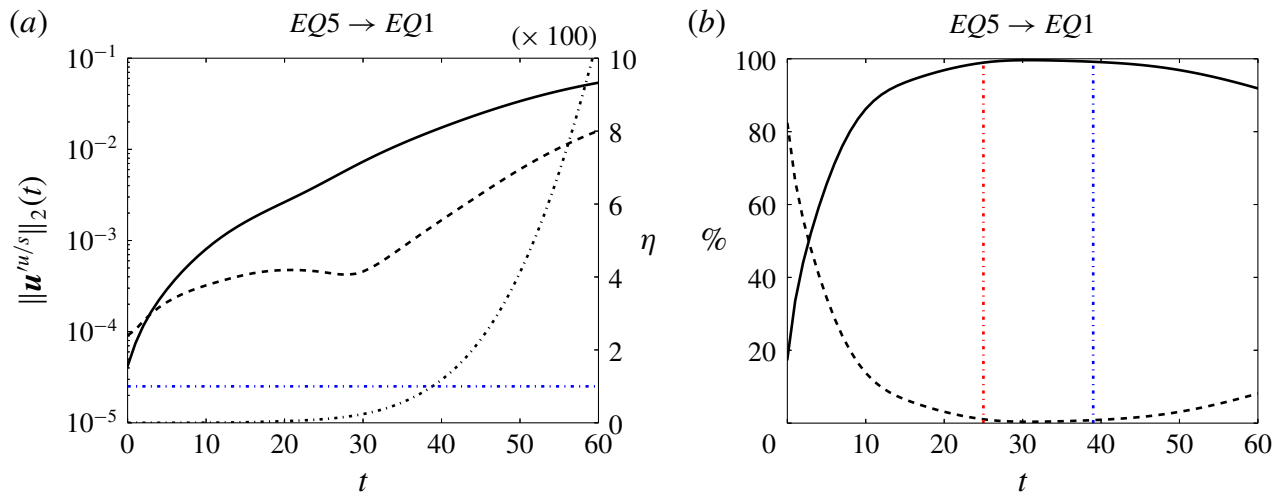

FIGURE 3. Time evolution of contributions in the stable and unstable subspaces of $E Q_{\text {out }}$ during the initial part of the trajectory $E Q 5 \rightarrow E Q 1$. (a) L2-norms $\left\|\boldsymbol{u}^{\prime s / u}\right\|_{2}$ of the projections onto the unstable ( $\boldsymbol{u}^{\prime}{ }^{u}$, solid line) and stable ( $\boldsymbol{u}^{\prime s}$, dashed line) subspace. The difference between the linearized and the full nonlinear evolution $\eta=$ $\left(\left\|\boldsymbol{u}_{\text {lin }}^{\prime}(t)-\boldsymbol{u}^{\prime}(t)\right\|_{2}^{2} /\left\|\boldsymbol{u}_{\text {lin }}^{\prime}(t)\right\|_{2}^{2}\right.$ ) (dotted line, right axis) characterizes the deviation of the invariant manifolds from the approximating linear subspaces due to manifold curvature in the direction of the trajectory. (b) Relative contribution measured in terms of relative energy in the unstable (solid line) and stable (dashed line) subspace. Vertical red dotted lines indicates when the relative contribution of the stable subspace $E^{s}(t) / E(t)$ drops below $1 \%$. Vertical blue dotted line indicates when the difference between the linearized and the full nonlinear evolution $\eta$ reaches $1 \%$.

For all identified orbits connecting finite energy shells of radius $r_{\text {out }}=10^{-4}, r_{\text {in }}=$ $10^{-3}$, the identified initial condition lies predominantly in the stable subspace of $E Q_{\text {out }}$, with relative energy in the stable versus unstable space ranging from $82 \%$ to $98 \%$. To follow the evolution of the contributions we decompose $\boldsymbol{u}^{\prime}(t)=\boldsymbol{u}^{\prime u}(t)+\boldsymbol{u}^{\prime s}(t)$ into components in the stable/unstable subspace. Figure 3(a) shows the time evolution of the L2-norm of the contributions within the stable/unstable subspace of the orbit while leaving $E Q_{\text {out }}$ for the connection $E Q 5 \rightarrow E Q 1$. Data for other connections is provided in the supplementary material available at https://doi.org/10.1017/jfm.2018.860. Both $\left\|\boldsymbol{u}^{\prime u}\right\|_{2}(t)$ and $\left\|\boldsymbol{u}^{\prime s}\right\|_{2}(t)$ initially increase. The growth of the stable contribution slows down and starts to decay, while in the unstable subspace sustained approximately exponential growth continues. If the dynamics were linear, we would expect the stable contribution to decay to zero. However, due to the curvature of the invariant manifolds, the projection on the stable subspace may grow again, when the stable subspace no longer well approximates the attracting curved stable manifold. This is evidenced by the subsequent growth of $\left\|\boldsymbol{u}^{\prime s}\right\|_{2}(t)$ starting at $t \approx 30$ when the deviation between linear and nonlinear evolution $\eta=\left(\left\|\boldsymbol{u}_{\text {lin }}^{\prime}(t)-\boldsymbol{u}^{\prime}(t)\right\|_{2}^{2} /\left\|\boldsymbol{u}_{\text {lin }}^{\prime}(t)\right\|_{2}^{2}\right)$ starts to increase. We thus observe transient growth in the stable subspace which is superseded by exponential growth along the unstable subspace. Consequently, the trajectory shows a transient excursion along the stable subspace, but eventually the orbit approaches the unstable subspace, as evidenced by the decaying relative contribution of the stable subspace (see figure $3 b$ ). This decay is observed during the initial part of the orbit and while the unstable subspace remains a good approximation of the unstable manifold describing the nonlinear dynamics (see vertical blue dotted line in figure $3 b$, and dotted line in figure $3 a$ ). For all connections found for a shell of radius $r_{\text {out }}=10^{-4}$ the contribution of $\boldsymbol{u}^{\prime}$ in terms of energy in the unstable subspace 


\section{Adjoints for computing heteroclinic orbits}

reaches more than $99 \%$ while the relative difference between the linear and nonlinear evolution $\eta$ remains bounded by $0.858 \%$. (Alternatively, when the ratio of L2-norms reaches $\left\|\boldsymbol{u}^{\prime \prime}\right\|_{2}>0.99\left\|\boldsymbol{u}^{\prime}\right\|_{2}, \sqrt{\eta}$ remains bounded by $7.23 \%$.) The analysis consisting of projecting onto the stable subspace and monitoring the deviation between linear and nonlinear time evolution thus strongly supports the following interpretation: during the initial part, the orbit identified by the optimization algorithm does not follow the unstable manifold of $E Q_{\text {out }}$, but rather exploits algebraic growth along transiently amplified directions predominantly in the stable manifold. However, after a short excursion along the stable manifold, the orbit approaches the unstable manifold and shadows a heteroclinic connection.

For two orbits $E Q 9 \rightarrow E Q 1$ and $E Q 11 \rightarrow E Q 1$ the passage time is too large for the optimization to converge if a small energy shell of radius $r_{\text {out }}=10^{-4}$ is chosen. For the connection $E Q 9 \rightarrow E Q 1$ and shell of radius $r_{\text {out }}=10^{-3}$, the contributions of $\boldsymbol{u}^{\prime}$ in terms of relative energy in the unstable subspace reach $99 \%$ while $\eta$ remains bounded by $0.763 \%$. For the orbit $E Q 11 \rightarrow E Q 1$ a strongly curved unstable manifold renders the linearization invalid after a short time and before the relative contribution in terms of energy (L2-norm) of the unstable manifold has reached 61\% (78\%). However, the contraction rate along the stable subspace is similar to that observed for all other orbits. This suggests that despite the importance of nonlinear effects the orbit follows the strongly curved unstable manifold when leaving $E Q_{\text {out }}$.

An analogous analysis of the dynamics linearized around $E Q_{i n}$ confirms that all orbits approach the final equilibria along their stable manifold. Consequently, the orbits connecting finite shells around two equilibria follow the unstable manifold of $E Q_{o u t}$ and the stable one of $E Q_{i n}$. The initial part of the orbit is affected by transient amplification in the stable manifold, but the excursion decays before an L2-distance from $E Q_{\text {out }}$ of order $10^{-2}-10^{-3}$ is reached. More importantly, this distance is small compared to the typical L2-norm of the distance between both connected equilibria. The ratio of the distance affected by transient amplification to distance between both equilibria ranges from 0.016 to 0.100 . Consequently, the orbit shadows the heteroclinic connections along at least $90 \%$ of the distance between equilibria. Data for the individual connections are given in the supplementary material.

We verify that the computed orbits are robust to changes of the size of the initial energy shell. All orbits available for $r_{\text {out }}=10^{-4}$ were successfully recomputed for a larger shell of radius $r_{\text {out }}=10^{-3}$. For connections where the dimension of the intersection of the unstable manifold of the initial equilibrium with the stable manifold of the final state is greater than one, e.g. $E Q 4 \rightarrow E Q 1$, there is a continuous family of connections between these two states. Consequently, varying the energy shell may yield slightly different connections within the continuum. Likewise, the constrained search in Halcrow et al. (2009) yields a specific connection, while the variational algorithm may find a different connection within the continuum. For all connections with one-dimensional intersection, independently of the energy shell, the algorithm always converges to the only existing connection. This is demonstrated in figure 4 for the orbit $E Q 4 \rightarrow E Q 3$.

\subsection{Connectivity of equilibrium states}

When visualizing the computed heteroclinic connections in the energy input $(I)$ versus dissipation rate $(D)$ plane (Kawahara \& Kida 2001) (figure 5a) one observes that most orbits are located in the vicinity of the edge state equilibrium $E Q 1$. With the exception of $E Q 10$ and $E Q 11$ all other connected equilibria have a lower dissipation than the 


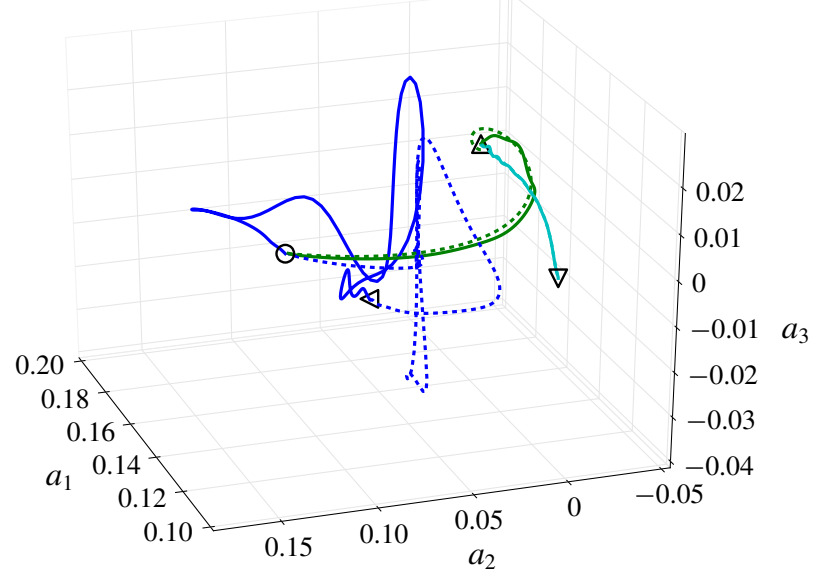

FIGURE 4. State-space projection (Gibson et al. 2008) of the orbit connecting $E Q 5 \rightarrow E Q 1$ (blue) and $E Q 4 \rightarrow E Q 1$ (green), for which the dimension of the intersection is three and two, respectively. For a specific connection, changing the energy shell around the initial equilibrium from $E_{0_{\text {out }}}=10^{-6}$ (solid line) to $E_{0_{\text {out }}}=10^{-8}$ (dashed line) results in finding different orbits. For those connections for which the dimension of the intersection is one, g.e. EQ4 $\rightarrow E Q 3$ (cyan), the algorithm finds the only existing connection.

average value $D=I \approx 2.5$ for long turbulent transients (Cherubini \& De Palma 2014). Along the majority of all connections - with exceptions $E Q 3 \rightarrow E Q 1, E Q 9 \rightarrow E Q 1$ - the friction Reynolds number $R e_{\tau}$ decreases, indicating that turbulent fluctuations are reduced along the orbit. This suggests that the presented heteroclinic connections are more relevant for relaminarization processes than the turbulent dynamics itself. Of special interest is the trajectory $E Q 11 \rightarrow E Q 1$, involving $E Q 11$ with dissipation well above the turbulent mean. This trajectory captures features of turbulent bursting.

To better visualize how the EQs are mutually connected, the heteroclinic connections are also plotted using the $3 \mathrm{D}$ orthonormal projection of the state space proposed by Gibson et al. (2008). The time series of the projection coefficients $\left(a_{1}(t), a_{2}(t), a_{3}(t)\right)$ are obtained using the inner product $a_{i}(t)=\left\langle\boldsymbol{u}(t), \boldsymbol{e}_{i}\right\rangle$ defined above, where $i=1,2,3$ and $\boldsymbol{e}_{i}$ are the orthonormal basis functions based on $E Q 2$ and its half-cell translated siblings (Gibson et al. 2008). The resulting trajectories projected onto this reduced basis are reported in figure $5(b)$. Within the network of heteroclinic connections, the edge state $E Q 1$ is centrally located. For all other equilibrium states there is a direct connection landing on $E Q 1$. This indicates that all the considered equilibria are states on the edge of chaos, characterizing the dynamics on the stable manifold of the edge state. This is consistent with the edge of chaos and the chaotic saddle being dynamically linked for the considered domain size and Reynolds number (Chantry \& Schneider 2014). The flow evolution along the newly constructed heteroclinic connections are discussed in the supplementary material, where we also provide flow visualizations for all connections (see the supplementary movie).

\section{Discussion and outlook}

In this work, we develop a variational method based on nonlinear minimization techniques to identify heteroclinic connections between equilibrium states. The variational method complements alternative shooting methods employed previously. 


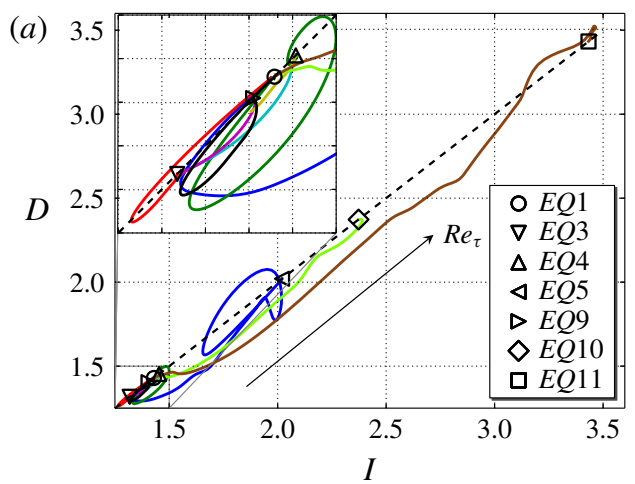

FIgURE 5. (a) Projection of the heteroclinic connections onto the energy input, $I$, and the dissipation rate, $D$, normalized by their value in the laminar flow. $(b)$ State-space projection onto the $3 \mathrm{D}$ orthonormal basis $\boldsymbol{a}_{1,2,3}$ of all the computed heteroclinic connections. Colours are the same as in figure 2.

A shooting method aims at finding an initial condition in the unstable manifold of the initial equilibrium that reaches the final one. This approach is only feasible for unstable manifolds of very low dimension to limit the search space, and also requires the manifold curvature to be negligible so that a linear combination of unstable eigenvectors approximates a perturbation of the initial equilibrium located in the unstable manifold. Specifically, Halcrow et al. (2009) consider perturbations spanned by only two specifically chosen unstable eigenvectors, which yields a one-parameter search problem. The alternative approach by Van Veen et al. (2011), based on work by Krauskopf et al. (2005), Krauskopf \& Osinga (2007), treats the search for heteroclinic connections as a boundary-value problem (BVP). The BVP is underdetermined, and a one-parameter family of solutions covering a part of the unstable manifold can be found by arclength continuation. This method is very accurate and robust, but it is limited to 2D unstable manifolds.

Compared to these alternative methods, the variational approach proposed here has several advantages: first, there is no constraint on the dimensionality of unstable manifolds and no ad hoc assumptions for a suitable reduced search space are necessary. Rather, the variational approach explores initial conditions within the full space. The proposed method allows one to approximate heteroclinic connections originating from states with multi-dimensional unstable manifold and thereby provides access to connections that cannot easily be identified with alternative methods. Three computed connections that do not land on the edge state $(E Q 4 \rightarrow E Q 3, E Q 9 \rightarrow E Q 3$ and $E Q 4 \rightarrow E Q 9)$ are examples of connections that are hard to identify using shooting methods, but can easily be found with the proposed variational method. Second, larger energy shells can be considered as there is no necessity for the unstable manifold to be well approximated by the unstable subspace which shooting methods constrain their search to.

While using a variational method to construct connections between finite energy shells removes constraints on the dimensionality of the unstable manifold of the initial equilibrium, the approach has some limitations. To avoid convergence issues the size of the energy shells should be sufficiently large to limit the transit time along the connection. Thereby the exponentially amplified dependence on initial conditions in the chaotic system can be controlled and the optimization problem remains smooth 


\section{Farano, S. Cherubini, J. C. Robinet, P. De Palma and T. M. Schneider}

enough for the iterative algorithm to converge. However, as a consequence of using finite energy shells, the algorithm may produce spurious results, since a connection between finite shells is not guaranteed to shadow a heteroclinic connection located within the intersection of the unstable manifold of the starting and the stable manifold of the final equilibrium. Consequently, a careful a posteriori analysis of the dynamics close to the two equilibria is an integral and unavoidable step to confirm that a computed orbit shadows a heteroclinic connections. Furthermore, due to transient growth effects, the initial part of the identified orbit may not shadow the heteroclinic connection. For all examples presented here, we observed this initial part to be short (less than $10 \%$ of the distance between equilibria) so that the constructed orbits between finite shells shadow heteroclinic connections along almost their full length.

In conclusion, the variational method has the disadvantage that a careful analysis of orbits between energy shell is needed to ensure that those approximate heteroclinic connections. However, the advantage is that the new method gives access to heteroclinic connections which cannot be easily computed using alternative techniques.

\section{Supplementary material and movie}

Supplementary material and a movie are available at https://doi.org/10.1017/jfm. 2018.860 .

\section{References}

Budanur, N. B., Short, K. Y., Farazmand, M., Willis, A. P. \& Cvitanović, P. 2017 Relative periodic orbits form the backbone of turbulent pipe flow. J. Fluid Mech. 833, 274-301.

ChAnTRY, M. \& SCHNEIDER, T. M 2014 Studying edge geometry in transiently turbulent shear flows. J. Fluid Mech. 747, 506-517.

Cherubini, S. \& De Palma, P. 2014 Minimal perturbations approaching the edge of chaos in a Couette flow. Fluid Dyn. Res. 46 (4), 041403.

Cherubini, S., De Palma, P., Robinet, J.-C. \& Bottaro, A. 2010 Rapid path to transition via nonlinear localized optimal perturbations in a boundary-layer flow. Phys. Rev. E 82 (6), 066302.

Crommelin, D. T. 2003 Regime transitions and heteroclinic connections in a barotropic atmosphere. J. Atmos. Sci. 60 (2), 229-246.

DonG, C. \& LAN, Y. 2014 A variational approach to connecting orbits in nonlinear dynamical systems. Phys. Lett. A 378 (9), 705-712.

Duguet, Y., Willis, A. P. \& Kerswell, R. R. 2008 Transition in pipe flow: the saddle structure on the boundary of turbulence. J. Fluid Mech. 613, 255-274.

Faisst, H. \& EcKhardt, B. 2003 Travelling waves in pipe flow. Phys. Rev. Lett. 91, 224502.

Farano, M., Cherubini, S., Robinet, J.-C. \& De Palma, P. 2015 Hairpin-like optimal perturbations in plane Poiseuille flow. J. Fluid Mech. 775, R2.

Farano, M, Cherubini, S, Robinet, J.-C. Robinet, De Palma, P. \& Schneider, T. M. 2018 How hairpin structures emerge from exact solutions of shear flows (in review).

Foures, DPG, CAUlfield, CP \& SCHMID, PJ 2013 Localization of flow structures using $\infty$-norm optimization. J. Fluid Mech. 729, 672-701.

Gibson, J. F. 2014 Channelflow: a spectral Navier-Stokes simulator in C++. Tech. Rep. U. New Hampshire, http://www.channelflow.ch.

Gibson, J. F., HAlCRow, J. \& CVitanović, P. 2008 Visualizing the geometry of state space in plane Couette flow. J. Fluid Mech. 611, 107-130.

Gibson, J. F., HAlcrow, J. \& Cvitanović, P. 2009 Equilibrium and traveling-wave solutions of plane Couette flow. J. Fluid Mech. 638, 243-266.

Halcrow, J., Gibson, J. F., Cvitanović, P. \& Viswanath, D. 2009 Heteroclinic connections in plane Couette flow. J. Fluid Mech. 621, 365-376. 


\section{Adjoints for computing heteroclinic orbits}

Hof, B. \& Budanur, N. B. 2017 Heteroclinic path to spatially localized chaos in pipe flow. J. Fluid Mech. 827, R1.

Hof, B., van Doorne, C. W. H., Westerweel, J., Nieuwstadt, F. T. M., Faisst, H., Eckhardt, B., Wedin, H., Kerswell, R. R. \& WalefFe, F. 2004 Experimental observation of nonlinear traveling waves in turbulent pipe flow. Science 305, 1594-1598.

Hopf, E. 1948 A mathematical example displaying features of turbulence. Commun. Pure Appl. Maths 1, 303-322.

Kawahara, G. \& KidA, S. 2001 Periodic motion embedded in plane Couette turbulence: regeneration cycle and burst. J. Fluid Mech. 449, 291-300.

Kawahara, G., Uhlmann, M. \& Van Veen, L. 2012 The significance of simple invariant solutions in turbulent flows. Annu. Rev. Fluid Mech. 44, 203-225.

Kerswell, R. R. 2018 Nonlinear nonmodal stability theory. Annu. Rev. Fluid Mech. 50 (1), 319-345.

Krauskopf, B.\& OsingA, H. M. 2007 Computing invariant manifolds via the continuation of orbit segments. In Numerical Continuation Methods for Dynamical Systems, pp. 117-154. Springer.

Krauskopf, B., Osinga, H. M., Doedel, E. J., Henderson, M. E., Guckenheimer, J., Vladimirsky, A., Dellnitz, M. \& Junge, O. 2005 A survey of methods for computing (un) stable manifolds of vector fields. Intl J. Bifurcation Chaos 15 (3), 763-791.

Kreilos, T., Veble, G., Schneider, T. M \& Eckhardt, B. 2013 Edge states for the turbulence transition in the asymptotic suction boundary layer. J. Fluid Mech. 726, 100-122.

LAN, Y. \& CVITANOVIĆ, P. 2004 Variational method for finding periodic orbits in a general flow. Phys. Rev. E 69 (1), 016217.

NagatA, M. 1990 Three-dimensional finite-amplitude solutions in plane Couette flow: bifurcation from infinity. J. Fluid Mech. 217, 519-527.

Pringle, C. C. T. \& Kerswell, R. R. 2010 Using nonlinear transient growth to construct the minimal seed for shear flow turbulence. Phys. Rev. Lett. 105, 154502.

Schneider, T. M., Gibson, J. F., Lagha, M., De Lillo, F. \& Eckhardt, B. 2008 Laminarturbulent boundary in plane Couette flow. Phys. Rev. E 78, 037301.

Suri, B., Tithof, J., Grigoriev, R. O. \& Schatz, M. F. 2017 Forecasting fluid flows using the geometry of turbulence. Phys. Rev. Lett. 118 (11), 114501.

Toh, S. \& Itano, T. 2003 A periodic-like solution in channel flow. J. Fluid Mech. 481, 67-76.

Van Veen, L. \& Kawahara, G. 2011 Homoclinic tangle on the edge of shear turbulence. Phys. Rev. Lett. 107 (11), 114501.

Van Veen, L., Kawahara, G. \& Atsushi, M. 2011 On matrix-free computation of 2D unstable manifolds. SIAM J. Sci. Comput. 33 (1), 25-44.

Viswanath, D. 2007 Recurrent motions within plane Couette turbulence. J. Fluid Mech. 580, 339-358.

WALEFFE, F. 2003 Homotopy of exact coherent structures in plane shear flows. Phys. Fluids 15 (6), $1517-1534$.

Wedin, H. \& KerSWell, R. R. 2004 Exact coherent structures in pipe flow: traveling wave solutions. J. Fluid Mech. 508, 333-371.

Willis, A. P., Cvitanović, P. \& Avila, M. 2013 Revealing the state space of turbulent pipe flow by symmetry reduction. J. Fluid Mech. 721, 514-540. 